\title{
Molecular Dynamics Simulation and Free Energy Calculation of Protein Arginine Methyltransferase 1
}

\author{
Xin Zhou \\ Beifang University of Nationalities, Yinchuan 750021, China \\ 1633289567@qq.com
}

\begin{abstract}
Protein arginine methyltransferase 1 (PRMT1) forms S-adenosylhomocysteine (SAH) and arginine by transferring methyl of SAM(S-adenosyl-L-methionine) to the substrate arginine, and then various effects in vivo occur. Our research group designed and synthesized 28 PRMT1 inhibitors which take furan ring as the mother nucleus in earlier stage, this research selected five representative inhibitors and composite of classical known PRMT1 inhibitor AMI1 and PRMT1 for 100 nanoseconds of molecular dynamics simulations to explore the binding modes of these compounds. The research found that these compounds stably bind to the PRMT1 pocket during the entire simulation process, but their binding modes are significantly different from AMI1. AMI1 occupies the binding site of arginine and amino acids, while the small molecule compound occupies the binding site of SAH. Then we used MMPBSA and Nmode to calculate the binding free energy of these small molecules and PRMT1, respectively, and found that the calculated value is positively correlated with the experimental value.
\end{abstract}

Keywords: PRMT1 inhibitor; molecular dynamics simulation; Gibbs free energy; molecular docking.

\section{Introduction}

Protein Arginine Methyltransferases (PRMTs) takes S-adenosyl-L-methionine (SAM) as a methyl donor; the methyl is catalyzed and transferred guanidyl nitrogen atom of protein arginine, and generate methylated arginine and the S-adenosyl-L-homocysteine (SAH). Protein Arginine Methyltransferases (PRMTs) participate in many biological processes, such as post-transcriptional modification of genes, RNA splicing and transport, cell signal transduction and embryonic development $[5,6]$ [7]. Type I PRMTs can further catalyze mMA into asymmetric dimethylarginine (ADMA), type II PRMTs can further catalyze mMA and the convert it to symmetric dimethylarginine (SDMA) [1]. PRMT1 is the major type I PRMT in mammals and it has been shown to be involved to the arginine methylation of more than $90 \%$ mammalian cells [8]. PRMT1 can affect various tumor types, such as breast cancer [9], liver cancer [10], prostate cancer [11], lung cancer [12], leukemia $[13,14]$, and colon cancer [15], these highlight their potential as important targets for drug development. The binding site of SAH consists of subsite 1 (adenosine binding site, consist of Phe44, Glu137, Val136 and Glu108, blue circle in Figure 1A) and subsite 3 (amino acid binding site, consist of Asp84, Gly86, Arg62 and Glu152 (Fig. 1A, yellow circle), Glu161, Tyr160, Tyr156, and Trp302 form the binding site of the substrate arginine (subsite 2 in Fig. 1B) (Fig. 1A red circle).

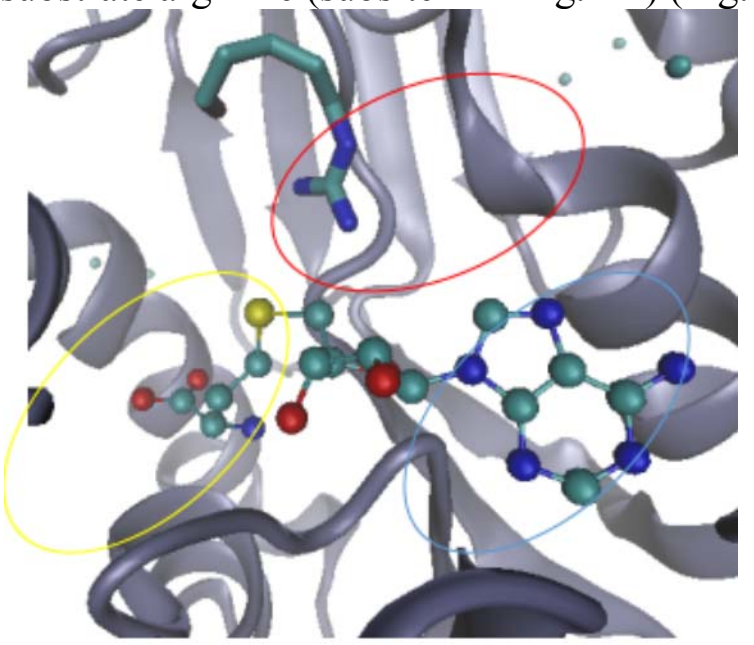

Figure 1 (A). Compound PRMT1 Active Site of SAH and Substrate Arginine 


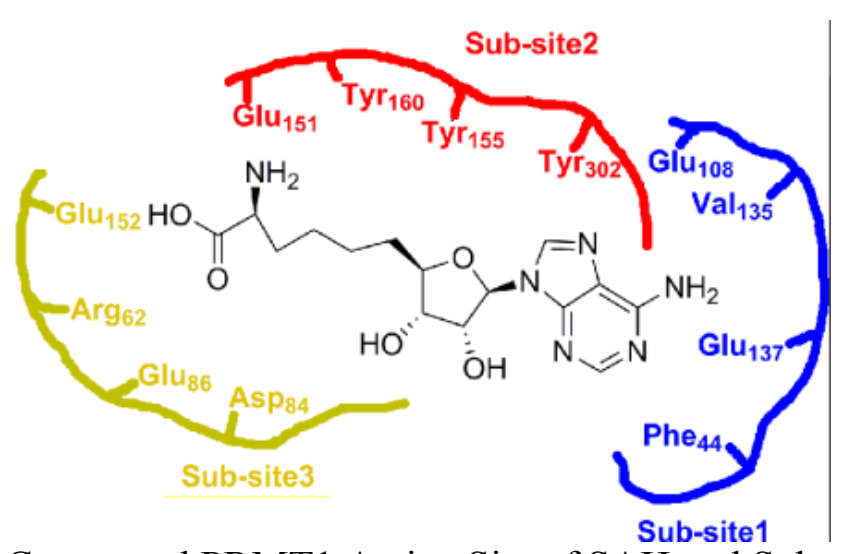

Figure 1 (B). Compound PRMT1 Active Site of SAH and Substrate Arginine
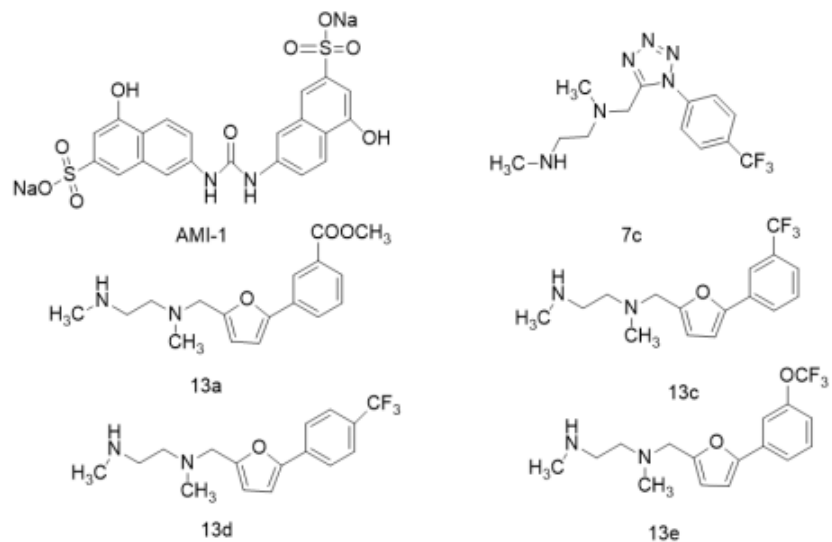

Figure 1 (C). Structure of 6 Active Molecules.

\section{Experimental Methods}

\subsection{Docking of GOLD to Active Molecules}

Every ligand molecules obtained by GOLD software was docked to the corresponding receptor protein; and ligand-binding pocket. The SAH and substrate arginine are contrasted with hPRMT1 (homologous model), by overlapping the crystal structure of rat PRMT3 (rPRMT3, PDB 1F3L) [2] and indicate its binding site (all protein atomic distances are within $5.0 \AA$ ), each ligand was docked 10 times by this software, random ligands from different orientation groups at each start, and the default automatic genetic algorithm parameter setting is used. Each compound molecule allows free rotation and the GoldScore scoring function to score, respectively, and find that each compound gives multiple docking conformations.

\subsection{Molecular Dynamics Simulation}

Molecular dynamics simulation uses the Amber Tools 16.0 program package. First, use the software's Antechamber module to fit the charge of small molecule. The tleap module was used to assign ff14Sf force field parameters and gaff force field parameters to hPRMT1 protein and small molecule ligands, respectively, and generate mop topology files for proteins, ligands, and composites. The composite system was simulated in the TIP3P water box environment, the minimum distance between the set protein and the box boundary was not less than $10 \mathrm{~nm}$, and the sodium ion was added to ensure the electrical neutrality of the entire system. The Shake algorithm is used to constrain the stretching vibration of hydrogen bond, periodic boundary conditions and particle mesh ewald (PME) methods is used to calculate long-range electrostatic effects, the non-bond interaction intercept is 0.8 $\mathrm{nm}$ and the simulation step size is $2 \mathrm{fs}$, when molecular dynamics simulations is conducted, first, the protein is constrained, 500 steps of steepest descent method and 500 steps of conjugate gradient 
method are used to optimize the energy for water molecules and ligands; then the system temperature is gradually increased from 0 to $300 \mathrm{~K}$ within $50 \mathrm{ps}$, and will remain in balance, and finally conduct $100 \mathrm{~ns}$ dynamic sampling analysis in the Isothemic-Iso-baric (NPT) mode at $300 \mathrm{~K}$ and $101 \mathrm{Kpa}$ constant temperature and pressure.

\subsection{Free Energy Calculation}

\subsubsection{MMPBSA Calculate Enthalpy Change}

Combined with calculation of free energy enthalpy and the entropy contribution, the perl scripts $\mathrm{mm} \_$pbsa.py and nmode modules in Amber 16 are completed, respectively, from the 100 ns trajectory, uniformly select the stable conformation of the 1000 frame molecular trajectory to calculate MMPBSA. First, the binding energy of the PRMT1 protein ligand complex was calculated, and then the binding energy of the stabilized protein ligand complex was calculated, the stable binding energy minus the initial binding energy, which was the enthalpy change of the active molecule.

\subsubsection{Interaction Calculate Entropy Change}

Take 1000 frames evenly from the 100ns trajectory and first calculate the residues (initial and stable amino acid binding sites) and entropy of ligands, the initial entropy value is subtracted from the stabilized entropy value, which is the entropy change of this active molecules.

\subsubsection{Interaction Calculate Free Energy}

Finally, the Gibbs free energy $\Delta \mathrm{G}$ is calculated with the formula $\Delta \mathrm{G}=\Delta \mathrm{H}-\mathrm{T} \Delta \mathrm{S}$.

\section{Experimental Calculations}

\subsection{Simulation Results of Molecular Dynamics}

First, six kinds of active molecules were docked with Gold software, and analyzed the molecular conformational intermolecular and intermolecular forces after docking, the dominant conformation was selected as the initial trajectory of molecular dynamics simulation. According to the molecular motion trajectory, it can be seen that there is less change in the whole active molecule trajectory of $13 \mathrm{a}, 13 \mathrm{c}, 13 \mathrm{~d}$, and 13e. The stable molecular conformation of the whole molecule mainly occupied the binding site of SAH, and the fluctuation is small, tends to be stable, and the stability is good, and AMI1 molecular trajectory fluctuation is great, and stability is poor, the molecular conformation of the molecular trajectory stability mainly occupy the binding sites of arginine and amino acid, and the molecular fluctuation is great; and then based on the calculation of free energy, it can be seen that the Gibbs free energy of 7c, 13a, 13c, 13d, 13e are $3.5091 \mathrm{KJ} / \mathrm{mol},-2.026 \mathrm{KJ} / \mathrm{mol},-5.1613 \mathrm{KJ} / \mathrm{mol}$, $7.1898 \mathrm{KJ} / \mathrm{mol}$, and $-3.6383 \mathrm{KJ} / \mathrm{mol}$ in order; the Gibbs free energy of AMI1 is $-0.5343 \mathrm{KJ} / \mathrm{mol}$.

\subsection{PRMT1 Molecular Dynamics Simulation of Six Active Molecules}

PRMT1 was used for molecular dynamics simulations with six active molecules, and the initial RMSD-Time diagram, average RMSD-Time diagram, and active molecular conformation diagram for the six active molecules at $100 \mathrm{~ns}$, as shown in Figure.2 RMSD-Time diagram (A), the mean RMSD-Time (B) diagram and the active molecular conformation diagram $(\mathrm{C})$, we can see that the RMSD diagram of the AMI1 active molecules vary widely and are molecularly unstable; both ends of the AMI1 molecule, amino acids Glu152, Arg62, Gly86, there is hydrogen bond interactions, The amino group at the other end of AMI-1 has hydrogen bonding with Phe44, Glu137 and Val136 residuesl. 


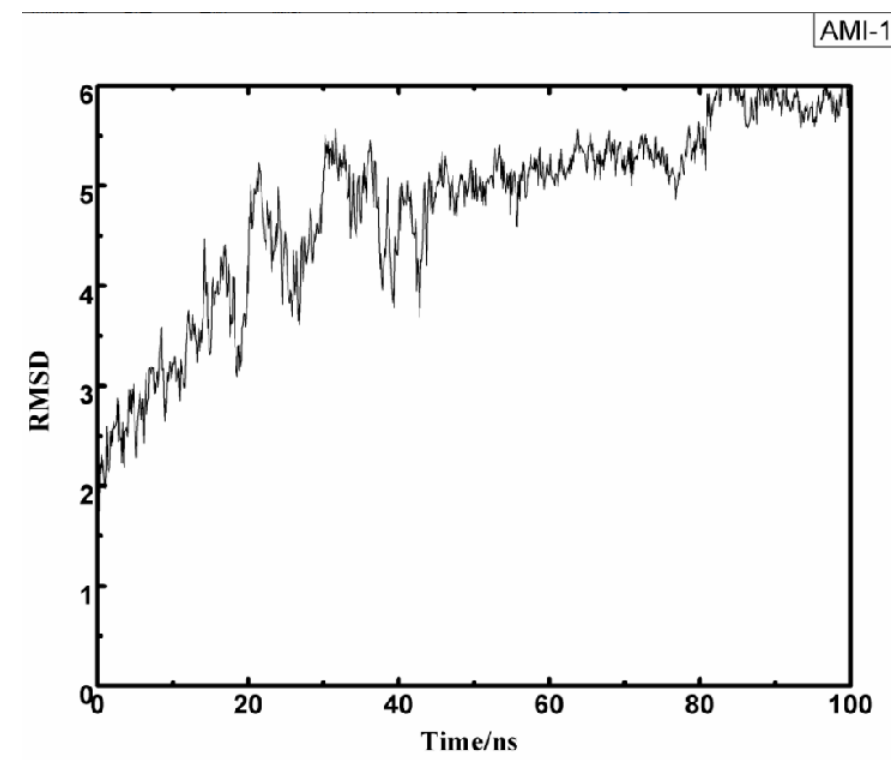

Figure 2 (A). Initial RMSD-Time Diagram

AMI-1

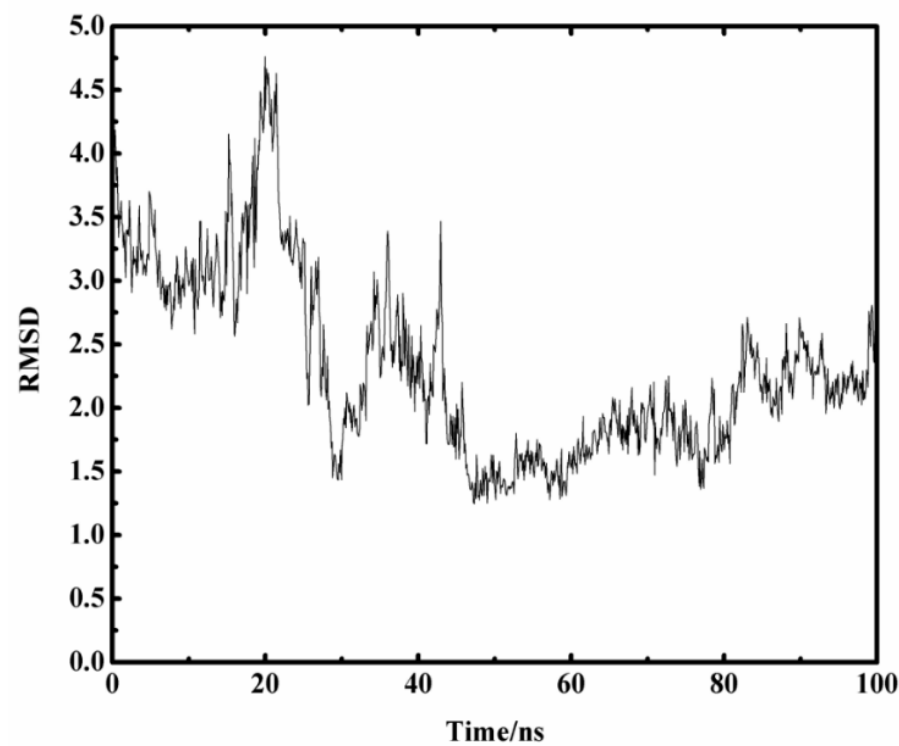

Figure 2 (B). Average RMSD-Time Diagram

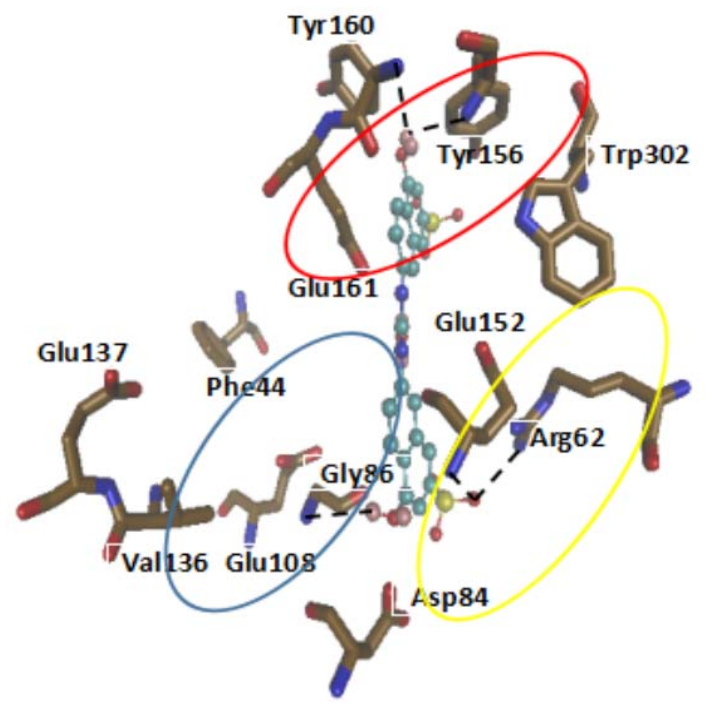

Figure $2(\mathrm{C})$. The Dominant Conformation of AMI1 
As shown in Figure 3, initial RMSD-Time diagram (A), mean RMSD-Time diagram (B), and active molecular conformation diagram (C), the RMSD diagram of the $13 \mathrm{~d}$ active molecule fluctuates smoothly, the trifluoromethyl of 13d molecule has hydrogen bonds with Val136 and Glu108 residues.

$13 \mathrm{~d}$

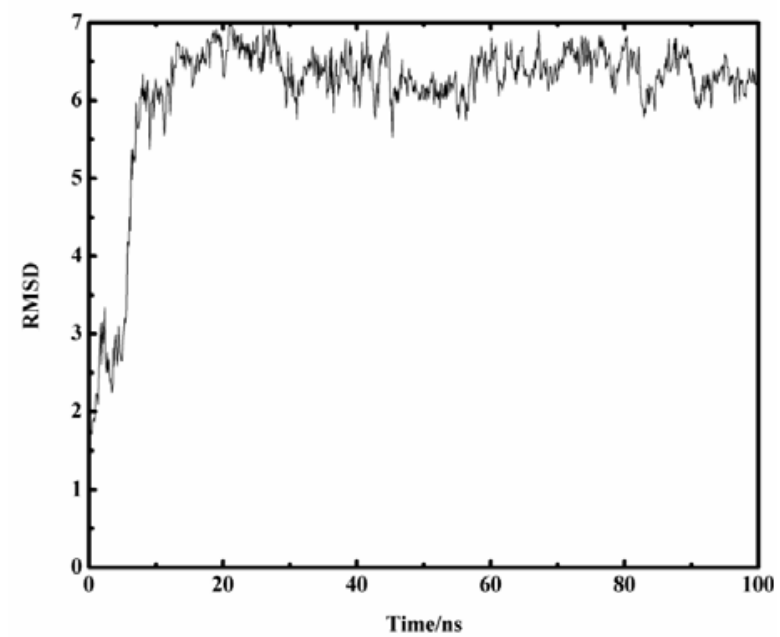

Figure 3 (A). Initial RMSD-Time Diagram

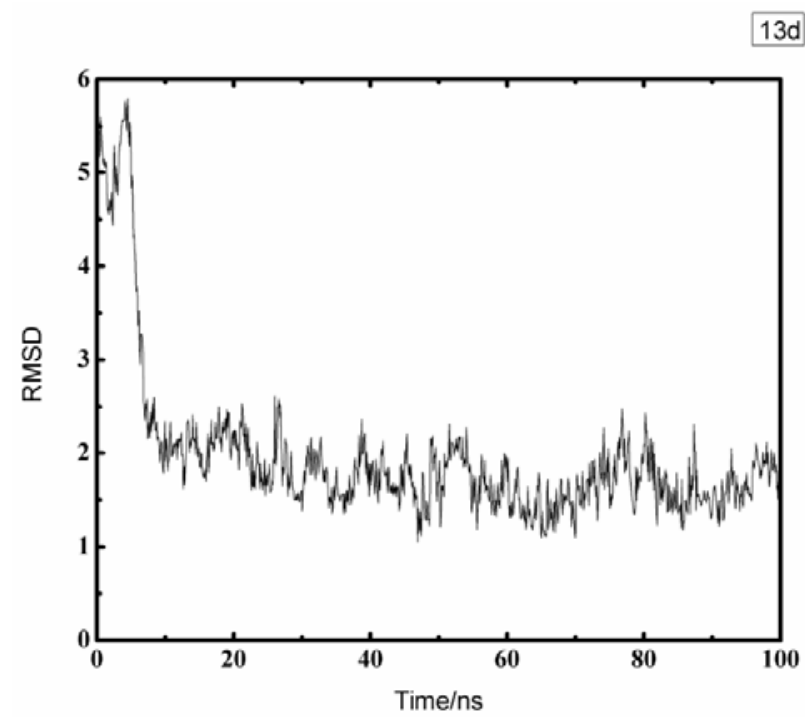

Figure 3 (B). Average RMSD-Time Diagram

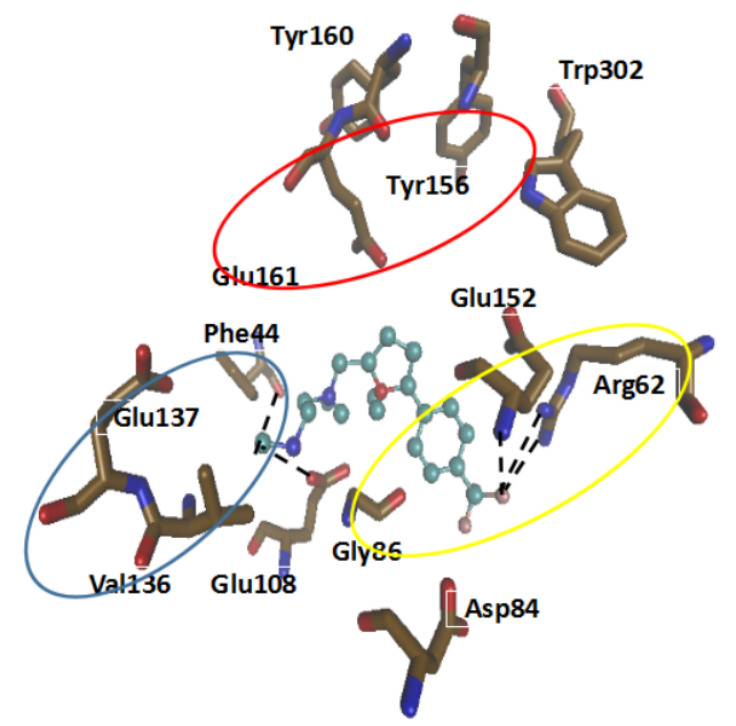

Figure 3 (C). The Dominant Conformation of 13d 


\section{Based on the Data Obtained From the Experiment, According to the Gibbs Free Energy Formula $\Delta \mathbf{G}=\Delta \mathbf{H}-\mathbf{T} \Delta \mathbf{S}$, the Theoretical Value of Binding Free Energy of the Active Molecule and Substrate PRMT1 is Calculated.Results and Discussion}

(1) the Gibbs free energy of AMI-1, 13a, 13c, 13e and 7c active molecules is also linearly related to the experimentally measured IC50, and the Gibbs free energy G of the compound molecule is proportional to IC50, namely, the smaller $\Delta \mathrm{G}$ is, the more stable the combination of the compound and PRMT1, the better the inhibitory activity. The results of molecular dynamics simulations are in agreement with the experimental bioactivity test results. We hope that the Gibbs free energy obtained by computer-aided calculations as a reference for the activity data of unknown compounds; it provides a theoretical basis for the design and synthesis of target compounds, and save the cost of molecular synthesis in the development of new drugs.

(2) The following analysis is conducted for the best active molecular conformation, atomic vibrations, and Gibbs free energy $\Delta \mathrm{G}$ : atomic vibrations of $13 \mathrm{~d}$ is within $10 \mathrm{~ns}-100 \mathrm{~ns}$, the molecular vibrations of initial RMSD, average RMSD, and potential energy function tend to stabilize. In the molecular dominant binding conformation of $13 \mathrm{~d}$, the trifluoromethyl docks and change into a blue circular region and forms a hydrogen bond with Phe44, while the ethylenediamine group occupies yellow circle area, and forms a strong electron interaction with Asp84 and Glu152. Effect. Due to the tight binding of the ethylenediamine group and Asp84, compound 13d can only change the trifluoromethyl orientation and occupy the binding site of Asp84 (yellow circles) and part of the substrate Arg sites (red circles).

\section{References}

[1]. BEDFORD M T. Arginine methylation at a glance [J]. Journal of Cell Science, 2007, 120(24): 4243-6.

[2]. BEDFORD M T, CLARKE S G. Protein arginine methylation in mammals: who, what, and why [J]. Molecular Cell, 2009, 33(1): 1-13.

[3]. LORENZO A D, BEDFORD M T. Histone arginine methylation [J]. Febs Letters, 2011, 585(13): 2024-31.

[4]. Gao Rui, Liu Lingxiao. Research Progress in protein arginine methylation transferase [J]. Shandong Medical Journal, 2014, 21): 104-6.

[5]. KRAUSE C D, YANG Z H, KIM Y S, et al. Protein arginine methyltransferases: evolution and assessment of their pharmacological and therapeutic potential [J]. Pharmacology \& Therapeutics, 2006, 113(1): 50-87.

[6]. SPANNHOFF A, SIPPL W, JUNG M. Cancer treatment of the future: Inhibitors of histone methyltransferases [J]. Int J Biochem Cell Biol, 2009, 41(1): 4-11.

[7]. LORENZO A D, BEDFORD M T. Histone arginine methylation. FEBS Lett [J]. Febs Letters, 2010, 585(13): 2024-31.

[8]. BEDFORD M T, RICHARD S. Arginine Methylation [J]. Molecular Cell, 2005.

[9]. GOULET I, GAUVIN G, BOISVENUE S, et al. Alternative splicing yields protein arginine methyltransferase 1 isoforms with distinct activity, substrate specificity, and subcellular localization [J]. Journal of Biological Chemistry, 2007, 282(45): 33009-21. 
[10]. LI B, LIU L, LI X, et al. miR-503 suppresses metastasis of hepatocellular carcinoma cell by targeting PRMT1 [J]. Biochem Biophys Res Commun, 2015, 464(4): 982-7.

[11]. WANG J, CHEN L, SINHA S H, et al. Pharmacophore-Based Virtual Screening and Biological Evaluation of Small Molecule Inhibitors for Protein Arginine Methylation [J]. Journal of Medicinal Chemistry, 2012, 55(18): 7978-87.

[12]. YOSHIMATSU M, TOYOKAWA G, HAYAMI S, et al. Dysregulation of PRMT1 and PRMT6, Type I arginine methyltransferases, is involved in various types of human cancers [J]. International Journal of Cancer Journal International Du Cancer, 2011, 128(3): 562-73.

[13]. CHEUNG N, CHAN L C, THOMPSON A, et al. Protein arginine-methyltransferasedependent oncogenesis [J]. Nature Cell Biology, 2007, 9(10): 1208-15.

[14]. SHIA W J, OKUMURA A J, YAN M, et al. PRMT1 interacts with AML1-ETO to promote its transcriptional activation and progenitor cell proliferative potential [J]. Blood, 2012, 119(21): 4953-62.

[15]. MATHIOUDAKI K, PAPADOKOSTOPOULOU A, SCORILAS A, et al. The PRMT1 gene expression pattern in colon cancer [J]. British Journal of Cancer, 2008, 99(12): 2094-9.

[16]. YANG H, OUYANG Y, MA H, et al. Design and synthesis of novel PRMT1 inhibitors and investigation of their binding preferences using molecular modelling [J]. Bioorganic \& Medicinal Chemistry Letters, 2017, 27(20).

[17]. ZHANG X, CHENG X. Structure of the Predominant Protein Arginine Methyltransferase PRMT1 and Analysis of Its Binding to Substrate Peptides [J]. Structure, 2003, 11(5): 509-20.

[18]. HONG W, LI J, LAUGHTON C A, et al. investigating the binding preferences of small molecule inhibitors of human protein arginine methyltransferase 1 using molecular modelling [J]. Journal of Molecular Graphics \& Modelling, 2014, 51(6): 193-202. 